\title{
Measurement of Gamma-ray Yield from Thick Carbon Target Irradiated by 5 and $9 \mathrm{MeV}$ Deuterons
}

\author{
Shouhei Araki ${ }^{1, *}$, Kazuhiro Kondo', Tadahiro Kin', Yukinobu Watanabe', Nobuhiro Shigyo², Kenshi Sagara ${ }^{3}$ \\ ${ }^{1}$ Department of Advanced Energy Engineering Science, Kyushu University, Fukuoka, Japan; ${ }^{2}$ Department of Applied Quantum Physics and Nuclear \\ Engineering, Kyushu University, Fukuoka, Japan; ${ }^{3}$ Department of Physics, Kyushu University, Fukuoka, Japan
}

\section{Original Research}

Received July 17, 2015

Revision August 31, 2016

Accepted November 28, 2016

Corresponding author: Shouhei Araki

Department of Advanced Energy Engineering Science, Kyushu University, Kasuga-koen 6-1, Kasuga, Fukuoka 8168580, Japan

Tel: $+81-92-583-7603$

Fax: $+81-92-583-7603$

E-mail:araki@aees.kyushu-u.ac.jp

This is an Open-Access article distributed under the terms of the Creative Commons Attribution NonCommercial License (http://creativecommons.org/ licenses/by-nc/4.0) which permits unrestricted noncommercial use, distribution, and reproduction in any medium, provided the original work is properly cited.

Copyright $\odot$ 2017The Korean Association for Radiation Protection

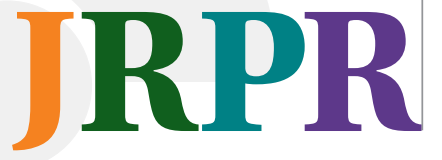

Background: The design of deuteron accelerator neutron source facilities requires reliable yield estimation of gamma-rays as well as neutrons from deuteron-induced reactions. We have so foar measured systematically double-differential thick target neutron yields (DDTTNYs) for carbon, aluminum, titanium, copper, niobium, and SUS304 targets. In the neutron data analysis, the events of gamma-rays taken simultaneously were treated as backgrounds. In the present work, we have re-analyzed the experimental data for a thick carbon target with particular attention to gamma-ray events.

Materials and Methods: Double-differential thick target gamma-ray yields from carbon irradiated by 5 and $9 \mathrm{MeV}$ deuterons were measured using an NE213 liquid organic scintillator at the Kyushu University Tandem accelerator Laboratory. The gamma-ray energy spectra were obtained by an unfolding method using FORIST code. The response functions of the NE213 detector were calculated by EGS5 incorporated in PHITS code.

Results and Discussion: The measured gamma-ray spectra show some pronounced peaks corresponding to gamma-ray transitions between discrete levels in residual nuclei, and the measured angular distributions are almost isotropic for both the incident energies.

Conclusion: PHITS calculations using INCL, GEM, and EBITEM models reproduce the spectral shapes and the angular distributions generally well, although they underestimate the absolute gamma-ray yields by about $20 \%$.

Keywords: Deuteron, Gamma-ray, NE213, Unfolding, PHITS

\section{Introduction}

A deuteron accelerator-based neutron source is considered as one of promising neutron sources in various neutron application fields such as production of radioisotopes for medical use [1], boron neutron capture therapy [2], fusion material irradiation test [3] and so on, because deuteron-induced reactions can produce high-intensity neutrons. In the design of deuteron accelerator facilities, it is important to estimate not only neutron but also gamma-ray yields from deuteron-induced reactions on the basis of experimental data and theoretical model calculations.

Up to now, we have measured systematically double-differential thick target neutron yields (DDTTNYs) for carbon, aluminum, titanium, copper, niobium, and SUS304 targets at two deuteron energies of 5 and $9 \mathrm{MeV}$ using an NE213 liquid organic scintillation detector [4-6]. Since the NE213 detector is also sensitive to gamma-rays, the data of gamma-rays events were taken simultaneously in the previous experiments. Howev- 
er, they were treated as background events in the neutron data analysis. As far as theoretical models are concerned, it was found that the intra-nuclear cascade of Liege (INCL) model [7] in PHITS [8] generally reproduce the DDTTNYs data of $\mathrm{Al}$ targets in both the shape and magnitude [9]. Also, it will be necessary to validate some reaction models used in PHITS using gamma-ray yield data for engineering design of deuteron accelerator facilities.

In the present work, we have re-analyzed the experimental data for a thick carbon target measured at two deuteron energies of 5 and $9 \mathrm{MeV}$ with particular attention to gamma-ray events, and derived double-differential thick target gammaray yields (DDTTGYs) by an unfolding method using FORIST code with NE213 response functions. The experimental results are compared with PHITS calculations for validation of the reaction models.

\section{Materials and Methods}

\section{Experiment}

Details of the experimental setup have been reported in Refs [4 - 6]. The experiment was carried out at the Kyushu University Tandem accelerator Laboratory (KUTL). A deuteron beam accelerated to 5 or $9 \mathrm{MeV}$ was injected on carbon foil of $0.5 \mathrm{~mm}$ in thickness which was set at the center of a compact vacuum chamber. The stopping length of $9 \mathrm{MeV}$ deuteron in carbon was calculated by SRIM code [10]. The target thickness was chosen so that $9 \mathrm{MeV}$ deuterons were stopped completely in the target. The chamber was isolated electrically from other experimental apparatus to measure the beam current.

Gamma-rays and neutrons emitted from the target were detected with an NE213 organic liquid scintillator $(5.08 \mathrm{~cm}$ in

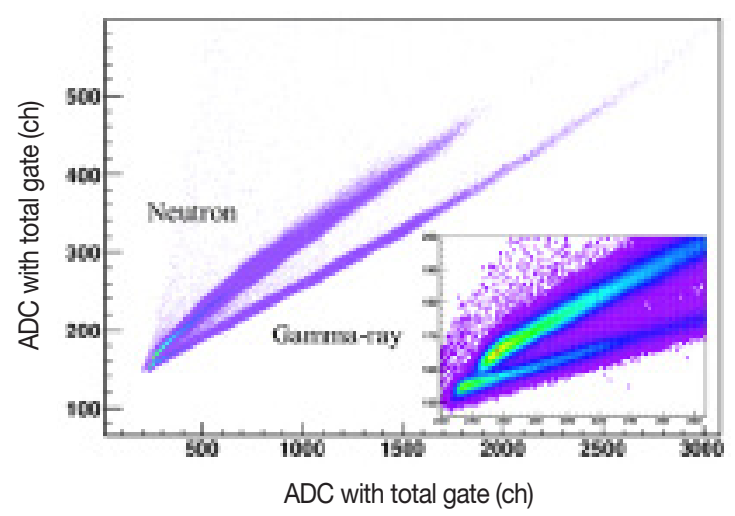

Fig. 1. Two dimensional plot of neutrons and gamma-rays discrimination using the two-gate integration method. diameter and $5.08 \mathrm{~cm}$ thick) optically coupled with a Hamamatsu H6410 photomultiplier (Hamamatsu Photonics K.K., Hamamatsu, Japan). The NE213 detector was set at eight angles $\left(0^{\circ}, 15^{\circ}, 30^{\circ}, 45^{\circ}, 60^{\circ}, 75^{\circ}, 90^{\circ}\right.$, and $\left.140^{\circ}\right)$ for $9 \mathrm{MeV}$ and at five angles $\left(0^{\circ}, 30^{\circ}, 60^{\circ}, 90^{\circ}\right.$, and $\left.140^{\circ}\right)$ for $5 \mathrm{MeV}$ with the distance of 1.6 to $2.4 \mathrm{~m}$ from the target position.

In order to estimate background gamma rays and neutrons, a separate measurement was made with placing an iron shadow bar (15 cm wide, $15 \mathrm{~cm}$ high, and $30 \mathrm{~cm}$ thick) between the target and the NE213 detector.

\section{Data analysis}

1) Gamma/Neutron discrimination

Both gamma-ray and neutron events detected by the NE213 detector were separated by using two-gate integration method [11]. Figure 1 shows a two-dimensional plot of total and slow components of the NE213 light outputs. Gammaray and neutron events were separated clearly even at low ADC channels corresponding to low light output.

\section{2) Light output calibration}

The measured light output spectra of gamma-ray events per ADC channel are converted to those per light output units of MeVee (MeV electron equivalent). For calibration of ADC channel to MeVee, the Compton edges of three standard gamma-ray sources, ${ }^{133} \mathrm{Ba}(0.36 \mathrm{MeV}),{ }^{137} \mathrm{Cs}(0.66 \mathrm{MeV})$, and ${ }^{60} \mathrm{Co}(1.17$ and $1.33 \mathrm{MeV}$ ) were used in the low light output region. The calibration in the high light output region was carried out by using the Compton edges of prompt gamma-rays from ${ }^{12} \mathrm{C}(\mathrm{d}, \mathrm{p}){ }^{13} \mathrm{C}(3.09 \mathrm{MeV}$ and $3.68 \mathrm{MeV})$ as shown in Figure 2. Additional calibration points in the higher region were given on the basis of the kinematics of the ${ }^{12} \mathrm{C}(\mathrm{d}, \mathrm{n}){ }^{13} \mathrm{~N}$

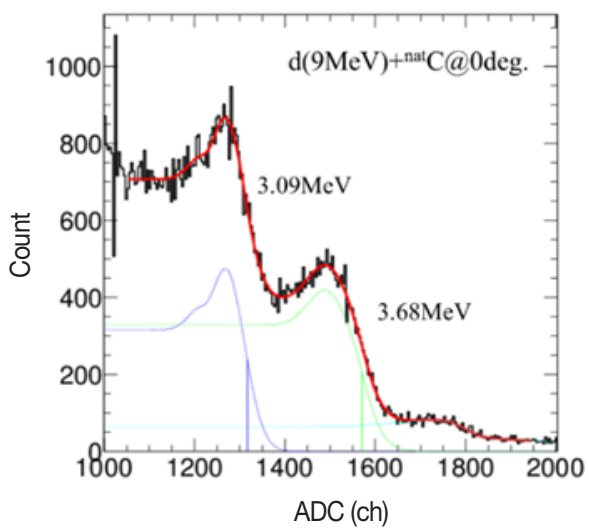

Fig. 2. Light output spectra of prompt gamma ray from thick carbon target at $0^{\circ}$ irradiated by $9 \mathrm{MeV}$ deuterons. 


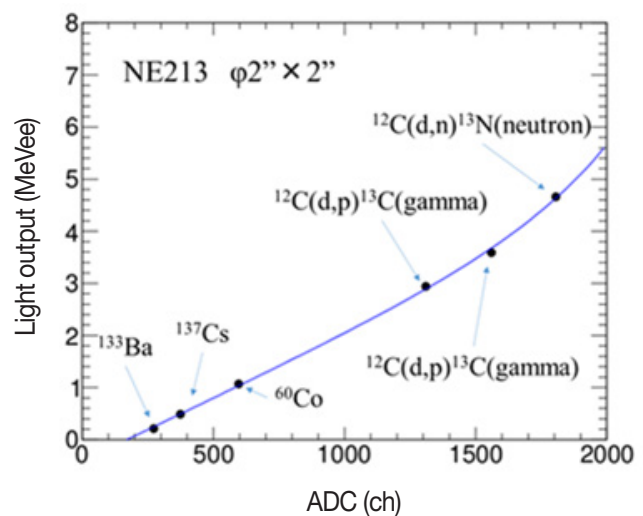

Fig. 3. Relationship between integrated charge amount given by ADC channel and light output in units of MeVee.

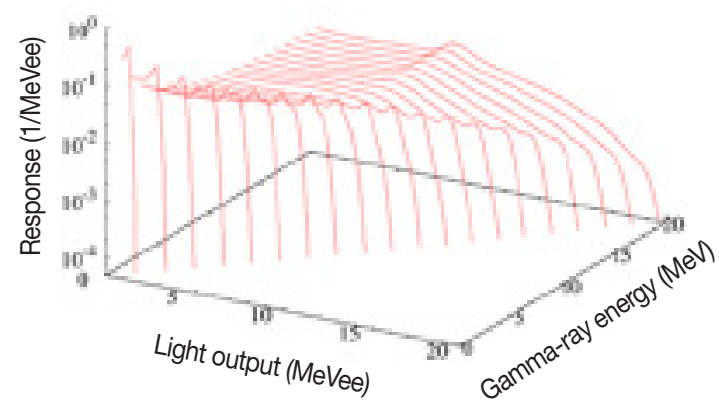

Fig. 4. Response functions of NE213 scintillator calculated by EGS code in PHITS.

reaction at $0^{\circ}$. The resultant calibration curve is shown in Figure 3.

\section{3) Response functions and unfolding}

The response functions necessary for unfolding of the measured light output spectra were calculated by EGS5 [12] incorporated with the PHITS code. The calculated response function was compared with the light output spectrum measured with a standard ${ }^{60} \mathrm{Co}$ source. Based on the comparison, the resolution of the detector itself was determined and the absolute efficiency was normalized to the measured result. The response functions after taking into account the resolution and normalization are shown in Figure 4.

Using the response functions, the unfolding of the measured light output spectra was performed by the FORIST code [13] based on the least squares method.

\section{Results and Discussion}

\section{Double-differential thick target gamma-ray yield}

The experimental DDTTGYs at $0^{\circ}$ from carbon irradiated by
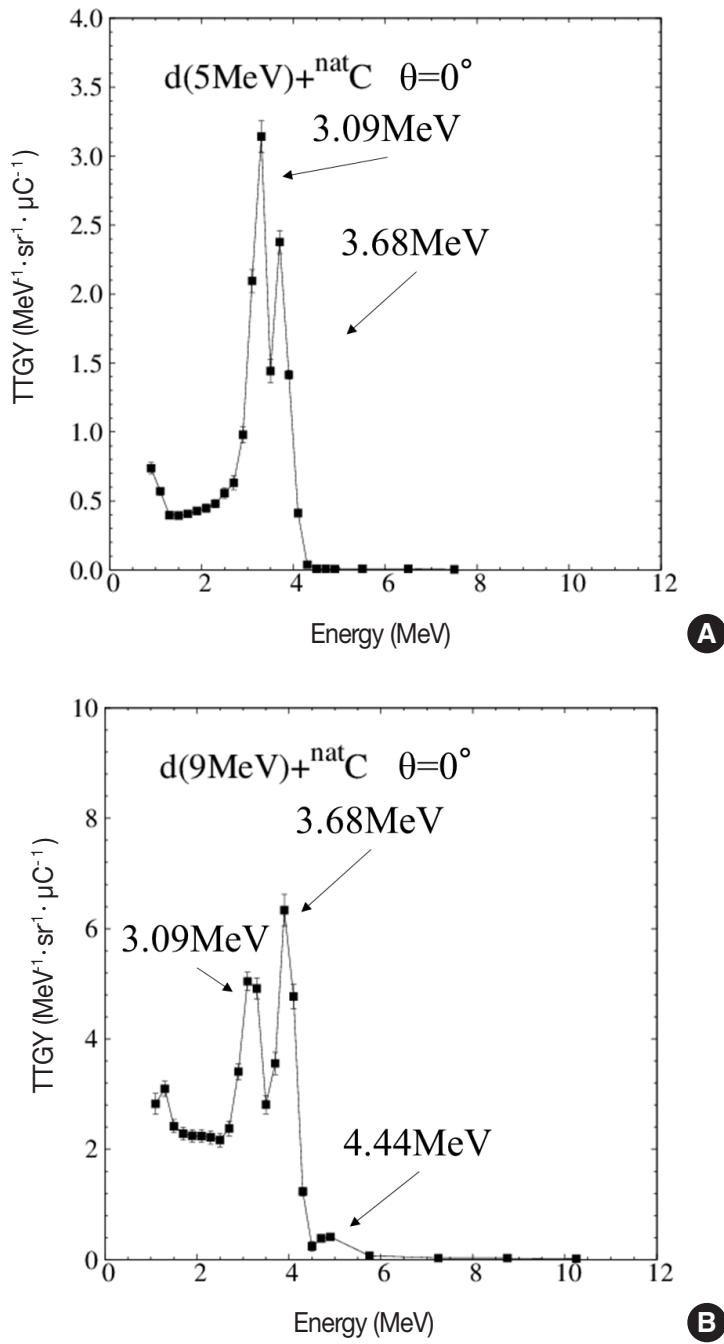

Fig. 5. Measured double-differential thick target gamma-ray yields at $0^{\circ}$ from carbon target irradiated by $5 \mathrm{MeV}(\mathrm{A})$ and $9 \mathrm{MeV}(\mathrm{B})$ deuterons. The solid lines are just for guiding the eye.

5 and $9 \mathrm{MeV}$ deuterons are shown in Figure 5. Two strong peaks corresponding to prompt gamma-rays from ${ }^{12} \mathrm{C}(\mathrm{d}, \mathrm{p})^{13} \mathrm{C}^{*}$ (3.09 MeV and $3.68 \mathrm{MeV}$ ) are clearly observed. In the panel (B), a weak peak corresponding to ${ }^{12} \mathrm{C}\left(\mathrm{d}, \mathrm{d}^{\prime}\right)^{12} \mathrm{C}^{*}(4.44 \mathrm{MeV})$ is also seen, while there is no appreciable peak in the panel (A) because the deuteron inelastic scattering at $5 \mathrm{MeV}$ is kinematically inhibited. It should be noted that the DDTTGYs measured at other angles have almost the same shape and magnitude as those at $0^{\circ}$ for both incident energies.

The error bars shown in Figure 5 include the statistical error and the uncertainty from the FORIST unfolding. Individual systematic errors were estimated in the similar way to Reference [6]: (a) determination of the solid angles and angular offset of the beam (3.2\%), (b) the uncertainty in the $n-\gamma$ 

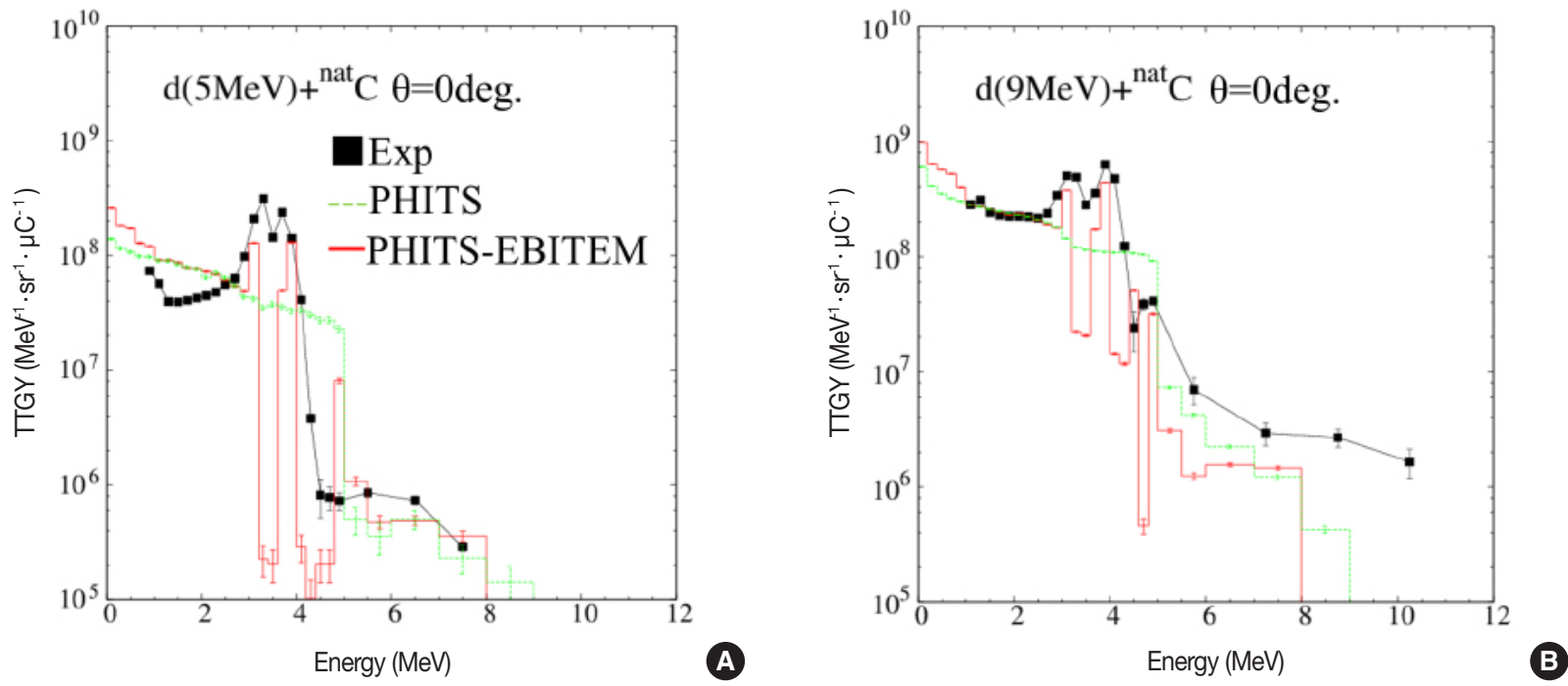

Fig. 6. Measured double-differential thick target gamma-ray yields at $0^{\circ}$ from carbon target irradiated by $5 \mathrm{MeV}(\mathrm{A})$ and $9 \mathrm{MeV}(\mathrm{B})$ deuterons compared with PHITS calculations using the simple nuclear de-excitation model and EBITEM. The solid line of experimental data is just for guiding the eye.

discrimination (5\%), (c) the accuracy of response function calculated by EGS in PHITS (18\%), and (d) the effect of gamma rays scattered by materials and air (2\%). The systematic error associated with the response functions was estimated based on the difference between the measured and calculated efficiencies for the standard 60Co source. The total systematic error amounts to be $19.1 \%$ by error propagation.

\section{Theoretical model analysis \\ 1) PHITS calculation}

The dynamical process and the subsequent evaporation process are described with the INCL model and the generalized evaporation model (GEM) [14], respectively, in PHITS calculation. The Shen formula [15] was chosen as a calculation option of total reaction cross section instead of the default option with the NASA [16] formula, because the former formula provided better result than the latter in our early work [2]. In addition, gamma-ray production reactions were calculated by the simple nuclear de-excitation model or the ENDSDF-Based Isomeric Transition and isomer production Model (EBITEM) [17] implemented in PHITS. The EBITEM can take into account the gamma-ray transition between dense levels in the high excitation energy region and sparse discrete levels in the low excitation energy region.

\section{2) Comparison with PHITS calculation}

Figure 6 shows comparisons of the DDTTGYs measured at $0^{\circ}$ for incident energies of 5 and $9 \mathrm{MeV}$ with PHITS calcula-

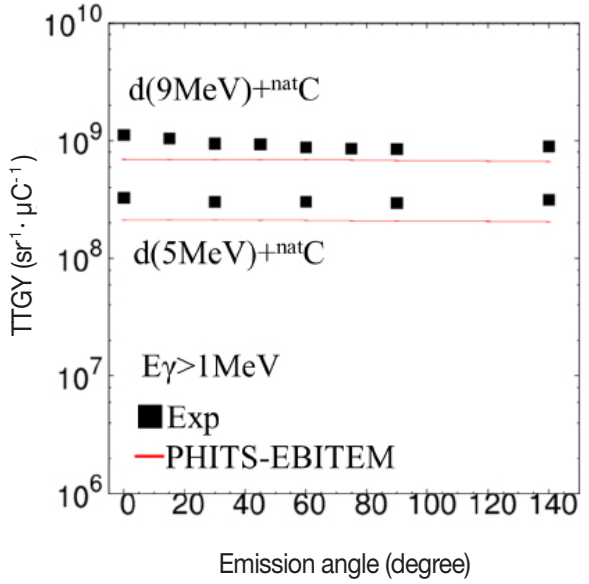

Fig. 7. Comparison of experimental gamma ray yields integrated over emission energy more than $1 \mathrm{MeV}$ with PHITS calculations using EBITEM.

tions. The PHITS calculations with EBITEM reproduce the peak structure seen in the measured DDTTGYs better than those with the simple nuclear de-excitation model. However, the experimental data show broader peak structure than the calculation with EBITEM. This may be because the present calculation cannot consider the experimental energy resolution properly.

Next, the experimental gamma-ray production yields integrated over the emission energy more than $1 \mathrm{MeV}$ are compared with the PHITS calculations using EBITEM in Figure 7. The PHITS calculations reproduce isotropic angular distribution observed in the measured data well, although under- 
estimation of about $20 \%$ is seen for both incident energies. The gamma-ray emission takes place mainly in the residual nuclei formed by particle emission as mentioned in Sec. 4.1. Underestimation of the particle emission may be associated with that seen in Figure 7. Further validation of the particle emission from deuteron-induced reactions on carbon in the PHITS calculation will be necessary to find out the cause of the underestimation seen in absolute gamma-ray yields.

\section{Conclusion}

The double-differential thick target gamma-ray yields from carbon irradiated by 5 and $9 \mathrm{MeV}$ deuterons were obtained over the wide angular range from $0^{\circ}$ to $140^{\circ}$ by re-analyzing the gamma-ray events taken in the previous measurement of thick target neutron yields performed with an NE213 scintillator. The unfolding method using the FORIST code was applied to the data analysis. The required response functions of NE213 scintillator were calculated by EGS5 incorporated in the PHITS code, and some corrections on detector resolution and absolute efficiency was made by comparison with the light output spectrum measured using a standard ${ }^{60} \mathrm{Co}$ source. Some pronounced peaks corresponding to gammaray transitions between discrete levels in residual nuclei were observed in the gamma-ray spectra measured for carbon. It was found that the PHITS calculation with EBITEM generally reproduces both the spectral shape and magnitude of the measured thick target gamma-ray yields. This encourages the use of EBITEM for estimation of gamma-ray yields in the design of deuteron accelerator-based neutron sources using PHITS.

\section{Acknowledgment}

The research was financially supported by the Sasakawa Scientific Research Grant from the Japan Science Society.

\section{References}

1. Nagai Y, et al. Generation of radioisotopes with accelerator neutrons by deuterons. J. Phys. Soc. Japan. 2013;82:064201.

2. Agosteoa S, Curzioc G, d'Erricoc F, Nath R, Tinti R. Characterization of an accelerator-based neutron source for BNCT versus beam energy. Nucl. Inst. Meth. A. 2002;476:106-112.

3. Moeslang A, Heinzel V, Matsui H, Sugimoto M. The IFMIF test facility design. Fusion, Eng, Des. 2006;81:863-871.

4. Shigyo N, et al. Measurement of deuteron induced thick target neutron yields at 9 MeV. J. Korean. Phys. Soc. 2010;59:1725-1728.

5. Tajiri Y, Watanabe Y, Shigyo N, Hirabayashi K, Nishizawa T, Sagara $\mathrm{K}$. Measurement of double differential neutron yields from thick carbon target irradiated by $5-\mathrm{MeV}$ and $9-\mathrm{MeV}$ deuterons. Prog. Nucl. Sci. Tech. 2014;4: 582-586.

6. Hirabayashi K, et al. Measurement of neutron yields from thick $\mathrm{Al}$ and SUS304 Targets Bombarded by $5-\mathrm{MeV}$ and $9-\mathrm{MeV}$ deuterons. Prog. Nucl. Sci. Tech. 2012;1:60-64.

7. Boudard A, Cugnon J, David JC, Leray S, Mancusi D. New potentialities of the Liège intranuclear cascade model for reactions induced by nucleons and light charged particles. Phys. Rev. C 2013;87:014606.

8. Sato T, et al. Particle and heavy ion transport code system PHITS, Version 2.52. J. Nucl. Sci. Technol. 2013;50(9):913-923.

9. Araki S, Watanabe Y, Kin T, Shigyo N, Sagara K. Measurement of double differential neutron yields from thick aluminum target irradiated by $9 \mathrm{MeV}$ deuteron. Energy Procedia 2015;71:197-204.

10. Ziegler JF, Biersack JP, and Littmark U. The stopping and range of Ions in solids. 2nd Ed. New York, NY. Pergamon Press. 2009; 95-128.

11. Moszynski M, et al. Identification of different reaction channels of high energy neutrons in liquid scintillators by the pulse shape discrimination method. Nucl. Phys. A. 1994;343:563-572.

12. Hirayama H, Namito Y, Bielajew AF, Wilderman SJ, Nelson WR. The EGS5 code system. KEK Report 2005-8. High Energy Accelerator Research Organization. 2005;8.

13. Johnson RH, Ingersoll DT, Wehring BW, Dorning JJ. NE-213 neutron spectrometry system for measurement from 1.0 to 20 MeV. Nucl. Inst. Meth. 1977;145:337-346.

14. Furihata S. Statistical analysis of light fragment production from medium energy proton-induced reactions. Nucl. Inst. and Meth. B. 2000;171:251-258.

15. Shen W, Wang B, Feng J, Zhan W, Zhu Y, Feng E. Total reaction cross section for heavy-ion collisions and its relation to the neutron excess degree of freedom. Nucl. Phys. A. 1989;491:135-146.

16. Tripathi RK, Cucinotta FA, Wilson JW. Accurate universal parameterization of absorption cross sections III-light system. Nucl. Inst. Meth. B. 1999;155:349-356.

17. Ogawa T, Hashimoto S, Sato T, Niita K. Development of gamma de-excitation model for prediction of prompt gamma-rays and isomer production based on energy-dependent level structure treatment. Nucl. Phys. B. 2014;325:35-42. 